\title{
HIGHLIGHTS
}

CELL BIOLOGY OF THE NEURON

\section{The travels of neuregulin}

Neuregulin- 1 and its erbB receptors are required for the formation of some synapses. The intracellular signalling function of erbB receptors, which are transmembrane tyrosine kinases, is well established. By contrast, it is not clear whether forms of neuregulin-1 that have an intracellular domain also have a signalling role inside the cells that express it. There was some evidence to suggest such a role, and now, data published by Bao et al. make a compelling case for neuregulin-1-mediated intracellular signalling.

One piece of information that pointed to a signalling role for neuregulin-1 was the fact that its absence in knockout mice led to death of the cells that would normally produce it. Bao et al. therefore tested whether exposing neuregulin-1expressing neurons to soluble erbB4 and erbB2, which together form a

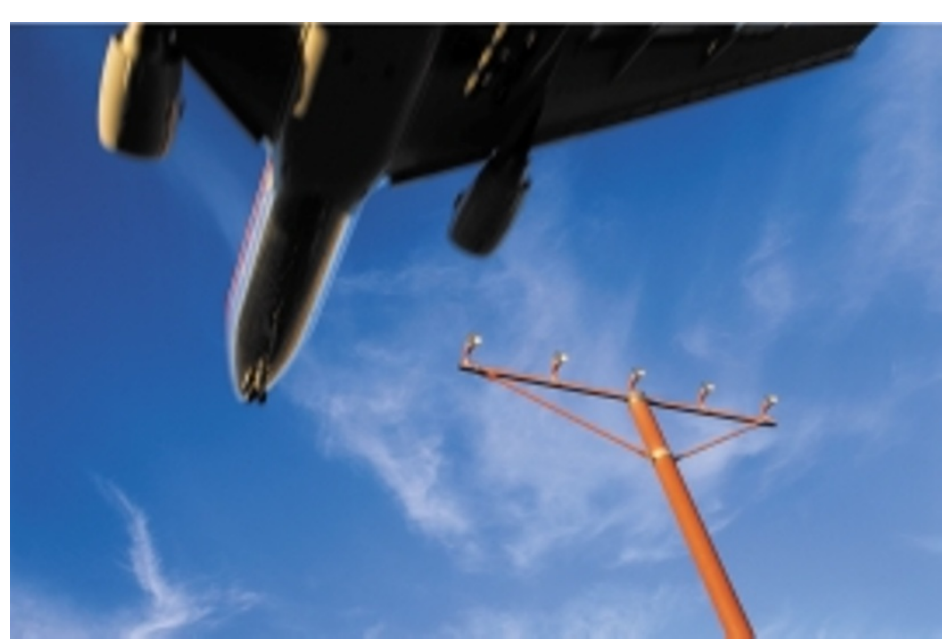

neuregulin-1 receptor, would be sufficient to increase cell survival. Indeed, the erbB receptors promoted neuronal survival, and this effect correlated with increased nuclear localization of the intracellular (but not the extracellular) domain of neuregulin1 and altered expression of several apoptosis-related genes.

A parsimonious explanation of these results is that the interaction of neuregulin-1 with erbB receptors 1 such that its intracellular domain travels to the nucleus and alters gene expression. Bao et al. looked for support for this model, and they found that the intracellular domain of neuregulin-1 has a nuclear localization signal, the absence of which prevents nuclear translocation of the protein. Moreover, by fusing the intracellular domain of neuregulin-1 to the DNA-binding domain of the leads to the proteolysis of neuregulin- transcription factor Gal4 and monitoring the expression of luciferase as a reporter, they found that neuregulin1 can act as a transcriptional transactivator. However, as neuregulin-1 lacks obvious DNA-binding and transcriptional-activator domains, it probably needs to interact with an unidentified protein to exert its effect on gene expression. Last, the authors obtained biochemical evidence of neuregulin-1 cleavage, and they showed by pharmacological means that $\gamma$-secretase, which is known to catalyze transmembrane proteolysis, is the relevant protease.

The bidirectional nature of the erbB-neuregulin-1 signalling system is reminiscent of what has been described for other molecules such as the ephrins and their Eph receptors. It would not be surprising if other similar bidirectional systems await discovery. In the meantime, we must add neuregulin- 1 to the rapidly growing list of molecules that are regulated by transmembrane proteolysis, an emerging theme in neuronal development and function.

Juan Carlos López and links ORIGINAL RESEARCH PAPER Bao, J. etal. Back signaling by the Nrg- 1 intracellular domain. J. Cell Biol. 161, 1133-1141 (2003)

FURTHER READING Sanes, J. R. \& Lichtman, J. W. Induction, assembly, maturation and maintenance of a postsynaptic apparatus. Nature Rev. Neurosci. 2, 791-805 (2001) 